\title{
The acquisition and transmission of vehicle traveling information to use smartphone
}

\author{
ZHU Huai-zhong \\ Shanghai Normal University Tianhua College, \\ Shanghai, China, 201815 \\ School of Transportation Engineering, Tongji \\ University, No.4800 Cao'an Road, Shanghai, \\ China, 201804; email: \\ zhuhuaizhong2000@163.com
}

\begin{abstract}
In recently, A massive research effort in monitoring vehicle and traffic conditions has gone into developing methodologies that can be used to offer value-added Information to managers and drivers. Such the process can be realized by the large popularity of smartphones and the sensor equipped in the vehicle. With supporting this purpose, in this paper a smartphone and cloud computing based platform is designed that the real-time vehicle information (such as velocity, acceleration, engine speed, road GPS, etc) is acquisition and transmit to the ITS center server. The vehicle traveling status is collect by On-Board Diagnostics (OBD) system. Then This paper In detail expound the OBD wireless Bluetooth communication with smartphone and cloud computing platform methods to realize the real-time data transmission to the server. Such necessary information can be widely used in cooperative vehicle and infrastructure system, traffic control system, intelligent transportation system and so on.
\end{abstract}

Keywords-Smartphone; Android; Bluetooth; Cloud computing; $\mathrm{OBD}$

\author{
YANG Xiao-guang3 \\ School of Transportation Engineering, \\ Tongji University, \\ Shanghai, China \\ email: yangxg@tongji.edu.cn
}

\section{INTRODUCTION}

The development of intelligence transportation system (ITS) enable the increased need for the theories, like traffic flow monitoring, signal control, cooperative vehicle and infrastructure, vehicle energy conservation and emissions reduction, etc. In those theories, the data collection is the focus, that is include engine speed, Tank humidity, Vehicle emissions, position and speed, etc. Traditionally, vehicle data collection have relied on the On-Board Diagnostics (OBD) system, connected with computer by COM. And this system could not adapt to the complex traveling conditions. Recently, Android smart phone and cloud computing promise to significantly improve the data collection. This paper propose an acquisition and transmission method of vehicle traveling information to use smart phones and OBD. Based on Android smart phone developed by Google and OBDII with the Bluetooth, the real time data is transmit to ITS center server using cloud computing. 


\section{SYSTEM ANALYSIS AND ARCHITECTURE} DESIGN

\section{A. System analysis}

In the tradition, the vehicle state measurement depend on the special . the vehicle information could be acquisition and transmission with Special vehicle terminal equipment. Because the vehicle terminal equipment is expensive and inconvenient to install and use in vehicle interior narrow space, their application has been limited largely. To overcame this disadvantages, we propose a novel solution by Android smartphone to get all the vehicle state information flexibly and easily instead of the vehicle terminal equipment. In Fig.1,

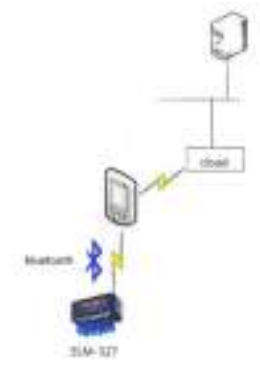

Figure 1.

The Android smartphone and OBDII hardware (ELM - 327) are connected by the Bluetooth. The Android Package (APK) programs running on the Android smartphone sent communication command to OBDII's Bluetooth, and receive OBDII's data according to the Bluetooth format, calculate the corresponding type of vehicle data. Then the calculated data upload to the ITS center server.

\section{B. Architecture design}

The system is composed of two parts, the one is Smartphone client program, and the other is ITS center server program. The Smartphone client program is designed to acquisition vehicle traveling information form OBD and transmission to ITS center in real time. The ITS center server program function is that receive the data transmitted via mobile phone and provide the data to other system(the vehicle speed for the cooperative vehicle and infrastructure system). In
Fig.2,

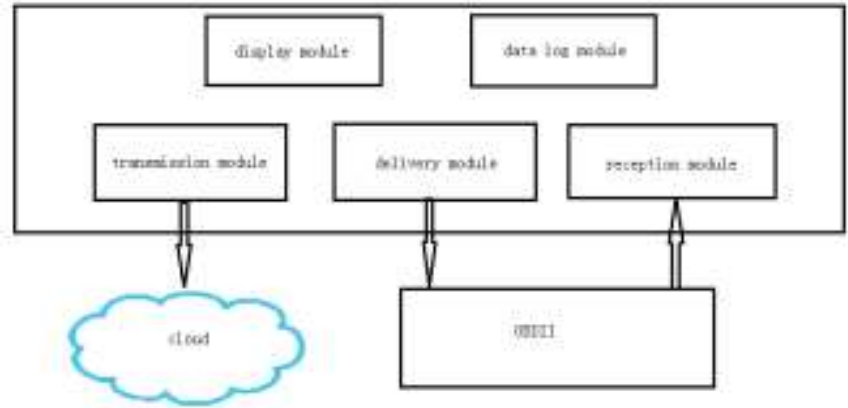

Figure 2.

The system architecture has three main modules: to deliver telegram to the OBD Bluetooth (delivery module), to receive telegram from the OBD Bluetooth (reception module), and to transmit telegram to the server (transmission module). In addition, The system also includes display module, data log module.

\section{SYSTEM ACHIEVING}

A. The Bluetooth communication principle

The Android smartphone and OBDII (ELM 327) involved in the system, that all have been equipped Bluetooth adapter. In smartphone Bluetooth is used as the client, and in OBDII is the server. In Fig.3,

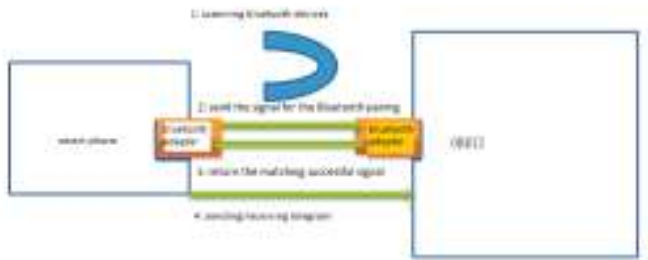

Figure 3.

Before communication, the process to establish a Bluetooth connection is that: (1) Smartphones scan Bluetooth devices around, and find the OBDII. (2) Smart phone sends the signal for the Bluetooth pairing, and OBD return the matching successful signal. Then the Bluetooth connection is established.

The process is achieved by use of android.bluetooth.BluetoothSocket. On the server side, use a BluetoothServerSocket to create a listening server socket. When a connection is accepted by the BluetoothServerSocket, it will 
return a new BluetoothSocket to manage the connection. On the client side, use a single BluetoothSocket to both initiate an outgoing connection and to manage the connection.

In practice, the most common type of Bluetooth socket is RFCOMM supported by the Android APIs. To create a connecting to a known device, the smartphone with the Android APIs firstly use

BluetoothDevice.createRfcommSocketToSer verRecord(), and then call connect() to attempt a connection to the device. This call will finish until a connection is established or the connection fails.

Once the socket is connected, whether initiated as a client or accepted as a server, open the IO streams by calling getInputStream and getOutputStream in order to retrieve java.io.InputStream and java.io.OutputStream objects, respectively, which are automatically connected to the socket.

Remind: Requires the android.Manifest.permission.BLUETOOTH permission.

B. Delivery module

After automatically connected with the OBDII Bluetooth, smartphone client program starts delivering telegram to OBD. In Fig.3, According to the demand, customer can check the sort of information through the Android smartphone interface. Then the demanded types of information is stored in the ArrayList, smartphone client program takes out the ArrayList data through the threads, and sends to the OBD Bluetooth.

Code1 Send telegram to OBD

private void runSenddataThread(final OutputStream outputStream) \{ new Thread() \{ public void run() \{

try \{ while (!mIsStop) \{

if

$($ mSendDataArrayList.size ()$>0)\{$

$$
\begin{aligned}
& =\text { mSendDataArrayList.get }(0) ; \\
& =\text { data.getBytes }() ;
\end{aligned}
$$

outputStream.write(bytes);

mSendDataArrayList.remove(0);

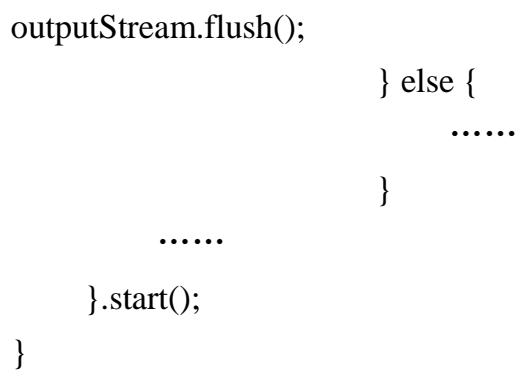

C. Reception module

Since telegram has been delivered to OBD Bluetooth, smartphone client program starts another thread in charge of receiving telegram from OBD Bluetooth. After receiving telegram, the smartphone analyzes it according to the Bluetooth communication format. Finally the analyzed data is stored in global variables. At the same time smartphone client program send a Message through the Handler to update the corresponding control content.

Code2 receive telegram from $\mathrm{OBD}$

private void runReadThread(final

DataInputStream inputStream) \{

new Thread() \{ public void run() \{ byte[] bytes; String rawData; try \{ while (!mIsStop) \{ byte $b=0$; rawData $=$ ""; while ((char) $(\mathrm{b}=$ (byte) inputStream.read()) != '>') \{

if ((char)

b != ' 
res.append((char) b);

\section{\}}

rawData =

res.toString().trim();

if

(rawData.contains("410D")) \{

// the

vehicle speed

m. what =

0x001;

Integer.parseInt(rawData.substring(

rawData.indexOf("410D") + 4,

rawData.indexOf("410D") + 6), 16);

Log.e("mSpeed", "" + mSpeed);

myHandler.sendMessage(m);

\} else if

(rawData.contains("410C")) \{ m.what =

0x002;

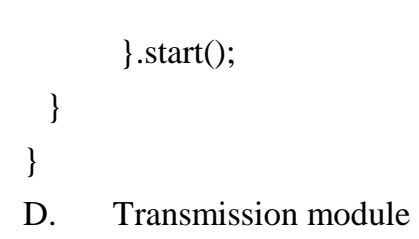

D. Transmission module

The OBD data collected by Smartphone client program is transmitted to ITS center server. The function of the module is implemented with the help of mobile Internet and cloud computing. With cloud computing, the mode of the connection to the server could be telecom communications network, also could be wifi, and other network. In transmit module, the Android WebView class is used.

\section{E. Display module}

The display module of Smartphone client program is responsible for the real-time display of the vehicle state information. This system uses thread to OBD Bluetooth to deliver telegram, In
Android, the thread could not directly update the content of activity interface control, so This module has to invoke the Handler, which sends the message to update the content of control.

Code3 display data

private Handler myHandler = new Handler() \{

@Override

public void handleMessage(Message

msg) \{

if $(\mathrm{msg}$. what $==0 \times 001)\{$

mSpeedTextView.setText("" + mSpeed); // to display the vehicle speed

$$
\}
$$

if $(\mathrm{msg}$. what $==0 \mathrm{x} 002)\{$

mEngineTextView.setText("' + mEngine);

// to display the engine speed

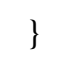

super.handleMessage(msg); \}

\}

F. Data log module.

In order to system testing, the OBD data write in smartphone data log files in real time, further to be encapsulated in the WriteLog class. Data $\log$ files are stored in SD, whose the directory appointed by the method in WriteLog classes.

G. System test and experiment

Before this system test, the smartphone was installed with the client program. The center server was installed with the server program. Additionally, the IP address of center server is provided.

This system is tested in two different conditions, (1) vehicle launch and not traveling: Android smartphone screen display as shown in Fig.4. 

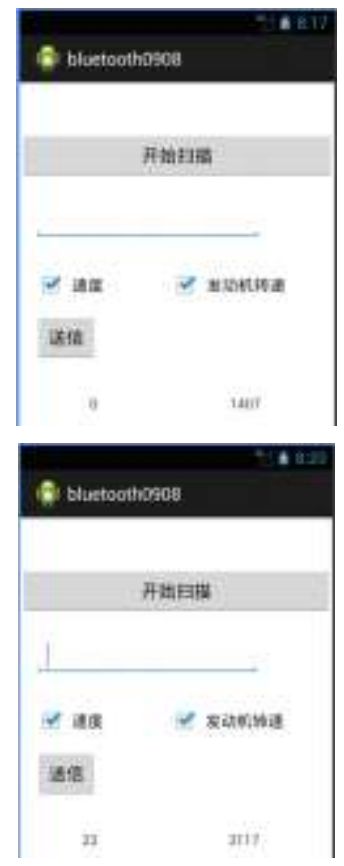

Figure 4.

Figure 5.

The vehicle speed is 0 , and the engine speed is 1400 RPM, consistent with the table data of vehicle cab. (2) vehicle launch and traveling, Android smartphone screen shown in Fig.5.

The vehicle speed is 20 , and the engine speed is 3100 RPM. These data sent to the ITS center server through the cloud computing platform, shown in Fig.6.

\begin{tabular}{|c|c|c|c|c|c|}
\hline \multicolumn{6}{|c|}{ 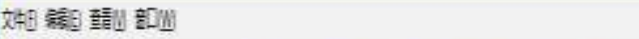 } \\
\hline \multicolumn{2}{|c|}{ 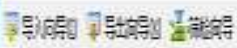 } & 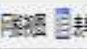 & 部首一 & & 影福 \\
\hline ialds & beet & gogreien: & teituast & & Seleaitor: \\
\hline Foran & & 1 & In & J & \\
\hline faten & & $\pi$ & 3117 & & \\
\hline
\end{tabular}

Figure 6.

By compared the vehicle travelling data with the vehicle dashboard data, the accuracy of data received via smartphone is quality which is the foundation of further analysis and utilized by ITS system.

\section{Conclusion and future study}

This paper describes the Android smartphone client program and server-side program build on cloud computing, which were applied to the collection of traveling data based on OBD. The smartphone client program send and receive data with OBDII, based on Bluetooth RFCOMM protocol system communication with OBD Bluetooth devices. The vehicle status information was transmitted to ITS center server with cloud computing. According to the results of the system test, the accuracy of the vehicle status data collected by system is quality. Moreover the system does not require expensive hardware to provides real-time, flexible status of vehicle. Due to the popularity of Android smartphone and stability of Bluetooth communication, the system is practical, high reliability, and easy to spread and expand, which can be widely used to monitor and control network traffic flow, cooperative vehicle and infrastructure system, and also available as the vehicle data source of car remote fault information management, vehicle monitoring system, and energy saving system.

\section{ACKNOWLEDGMENTS}

This research was made possible through funding from the Ministry of Science and Technology of the People's Republic of China under Grant 2012AA112306.

\section{REFERENCES}

[1] J. Zaldivar, C. T. Calafate, J. C. Cano, P. Manzoni, Providing Accident Detection in Vehicular Networks Through OBD-II Devices and Android-based Smartphones, 5th IEEE Workshop On User MObility and VEhicular Networks, Bonn, Germany, 2011.

[2] Hilpert H, Thoroe L, Schumann M (2011) Real-time data collection for product carbon footprints in transportation processes based on OBD2 and smartphones. In: Proc44th Hawaii int conf on system science(HICSS 44), pp 1-10

[3] C. Shi, H. J. Lee, J. Kurczal, and A. Lee. Routine Driving Infotainment App: Gamification of Performance Driving. In Adjunct Proceedings of the 4th International Conference on Automotive User Interfaces and Interactive Vehicular Applications, pages 181-183, Oct. 2012. 
[4] C. Campolo et al., SMaRTCaR: an integrated smartphone-based platform to support traffic management applications, in: First International Workshop on Vehicular Traffic Management for

Smart Cities (VTM), 2012.

[5] Fantesini L, Ferretti M, Marzani S, et al. Smartphones and tablets integration into agricultural and forestry machineries[C]//Information Technology, Automation and Precision Farming. International Conference of Agricultural Engineering-CIGR-AgEng 2012: Agriculture and Engineering for a Helathier Life, Valencia, Spain, 8-12 July 2012. CIGR-EurAgEng, 2012: C-2134.

[6] Hu X, Leung V, Li K G, et al. Social drive: a crowdsourcing-based vehicular social networking system for green transportation[C]//Proceedings of the third ACM international symposium on Design and analysis of intelligent vehicular networks and applications. ACM, 2013: 85-92.

[7] Torque Pro. available at https://play.google.com/.

[8] Wilfinger D, Murer M, Baumgartner A, et al. The car data toolkit: smartphone supported automotive $\mathrm{HCI}$ research[C]//Proceedings of the 5th International Conference on Automotive User Interfaces and Interactive Vehicular Applications. ACM, 2013: 168-175.

[9] U. Hernandez, A. Perallos, N. Sainz, and I. Angulo, " Vehicle on board platform: Communications test and prototyping, ” in Intelligent Vehicles Symposium (IV), 2010 IEEE, pp. $967-972,2010$.

[10] M.-C. Chen, J.-L. Chen, and T.-W. Chang, " Android/OSGi-based vehicular network management system, ” Elsevier Computer Communications, vol. 34, no. 2, pp. 169 - 183, 2011.

[11] Tarik Al-Ani, Tony Savarimuthu, and Maryam Purvis, “ Android-based in-vehicle infotainment system (aivi), ” in Information Science Postgraduate Day 2010, (Dunedin, New Zealand), pp. 51 - 52, October 2010.
[12] Y.-H. Cheng, W.-K. Kuo, and S.-L. Su, “An android system design and implementation for telematics services, ” in Intelligent Computing and Intelligent Systems (ICIS), 2010 IEEE International Conference on,vol. 2, pp. 206 $-210,2010$.

[13] C. Spelta, V. Manzoni, A. Corti, A. Goggi, and S. Savaresi, "Smartphone-based vehicle-to-driver/environment interaction system for motorcycles," Embedded Systems Letters, IEEE, vol. 2, no. 2, pp. 39 - 42, 2010.

[14] Lin, J. Y., Chen,S. C., Shih, Y. T., et al. “A Study on Remote On-Line Diagnostic System for Vehicles by Integrating the Technology of OBD, GPS, and 3G. " World Academy of Science, Engineering and Technology, August 2009, (56): 435-441

[15] Chen, S. H., Wei, Y. R. (2010), “A study on speech control interface for vehicle on-board diagnostic system." Proceedings - 4th International Conference on Genetic and Evolutionary Computing, Shenzhen, China, ICGEC 2010, 614-617.

[16] V. C. Magaa and M. M.Organero, "Artemisa: Using an Android device as an Eco-Driving assistant, ” IEEE International Conference on Consumer Electronics, ICCE, 2011. 